\title{
FIXED POINTS OF ANOSOV MAPS OF CERTAIN MANIFOLDS
}

\author{
JONATHAN D. SONDOW
}

\begin{abstract}
Lemma. If $H$ is a graded exterior algebra on odd generators with augmentation ideal $J$ and $h: H \rightarrow H$ is an algebra homomorphism inducing $J / J^{2} \rightarrow J / J^{2}$ with eigenvalues $\left\{\lambda_{i}\right\}$, then the Lefschetz number $L(h)=$ $\Pi\left(1-\lambda_{i}\right)$. The lemma is applied to an Anosov map or diffeomorphism of a compact manifold with real cohomology $H$ to give sufficient conditions that none of the eigenvalues $\lambda_{i}$ be a root of unity and that there exist a fixed point. In particular, every Anosov diffeomorphism of a compact connected Lie group has a fixed point.
\end{abstract}

1. Introduction and statement of results. An Anosov map of a smooth manifold $M$ is a smooth map $f: M \rightarrow M$ such that (1) There is a splitting of the tangent bundle $T(M)$ into a continuous Whitney sum $T(M)=E^{s}+E^{u}$ of subbundles which is invariant under the derivative map $D f: T(M) \rightarrow$ $T(M)$.

(2) There exists a Riemannian metric \|\| on $T(M)$ and constants $C>0$, $C^{\prime}>0,0<\lambda<1$, such that

$$
\left\|D f^{m}(v)\right\| \leqslant C \lambda^{m}\|v\| \text { and }\left\|D F^{m}(w)\right\| \geqslant C \lambda^{\prime} \lambda^{-m}\|w\|
$$

for all $v \in E^{s}, w \in E^{u}$, and $m \in Z^{+}$. One checks easily (see [9, §3.1]) that for $M$ compact the second condition is independent of which Riemannian metric is chosen.

An Anosov diffeomorphism is an Anosov map which is a diffeomorphism. Examples on the $n$-torus $T^{n}=S^{1} \times \ldots \times S^{1}$ are gotten by taking a matrix $f_{0} \in G L(n, Z)$ none of whose eigenvalues has absolute value 1 . Then $f_{0}$ induces an automorphism $f$ of $T^{n}=R^{n} / Z^{n}$ which, it is not hard to show, is an Anosov diffeomorphism. Nontoral examples have been given by Smale [9, $\S 1.3]$ on nilmanifolds and by Shub [8, p. 189] on infranilmanifolds.

Examples of Anosov maps $g$ can be constructed on products $M \times N \times P$ by taking $g=p \circ\left(\operatorname{id}_{M} \times f \times e\right)$, where $p: M \times N \times P \rightarrow * \times N \times P \subset M$ $\times N \times P$ is projection, $f: N \rightarrow N$ is an Anosov diffeomorphism, and $e$ : $P \rightarrow P$ is an expanding map (= Anosov map with $E^{s}=0$; see Shub [8]).

In $[9, \S 3.4]$, Smale asks whether every Anosov diffeomorphism has a fixed

Presented to the Society, January 26, 1970; received by the editors January 26, 1970 and, in revised form, May 6, 1976.

AMS (MOS) subject classifications (1970). Primary 58F15, 58F20; Secondary 15A75.

Key words and phrases. Anosov diffeomorphism, periodic point, exterior algebra, Lie group, Lefschetz number, eigenvalue, root of unity. 
point. We obtain the following partial answers. Say that an algebra $H$ over $R$ is a $T$-algebra if $H$ is isomorphic to the real cohomology algebra of a product of odd-dimensional spheres; i.e., $H$ is a graded exterior algebra on generators of odd degree. A manifold whose real cohomology is a $T$-algebra will be called a $T$-manifold.

THeOREM 1. Let $f$ be an Anosov map of a compact T-manifold $M$. If $E^{u}$ is orientable and $f$ has a periodic point then $f$ has a fixed point.

Theorem 2. If $M$ is a compact T-manifold, then every Anosov diffeomorphism of $M$ with $E^{s}$ or $E^{u}$ orientable has a fixed point.

COROllaRY. Every Anosov diffeomorphism of a compact, connected Lie group $G$ or of $G / F, F$ a finite subgroup of $G$, has a fixed point.

This generalizes the case $G=T^{n}$ proved by Franks [3] and Manning [7].

The following result, which res:ricts the homotopy classes that admit Anosov diffeomorphisms, is also an extension of work of Franks [3] on tori and Manning [6], [7] on infranilmanifolds. See also Hirsch [4].

TheOREM 3. Let $f$ be an Anosov diffeomorphism of a compact T-manifold $M$, with $E^{u}$ or $E^{s}$ orientable. Denote by $J$ the augmentation ideal of $H^{*}(M ; R)$ consisting of positive dimensional elements. Then no eigenvalue of $\overline{f^{*}}: J / J^{2} \rightarrow$ $J / J^{2}$ is a root of unity.

The main tool used in the proofs of these results is the following algebraic lemma, which may be of independent interest.

Lemma. Let $H$ be a T-algebra with augmentation ideal $J$ and let $h: H \rightarrow H$ be an algebra homomorphism inducing $J / J^{2} \rightarrow J / J^{2}$ with eigenvalues $\left\{\lambda_{i}\right\}$. Then the Lefschetz number $L\left(h^{q}\right)=\Pi_{i}\left(1-\lambda_{i}^{q}\right)$ for $q>0$.

Recall the definition

$$
L(h)=\sum_{k=0}^{n}(-1)^{k} \operatorname{trace}\left(h \mid H_{k}: H_{k} \rightarrow H_{k}\right),
$$

where $H=H_{0} \oplus H_{1} \oplus \ldots \oplus H_{n}$ is the grading of $H$.

I do not know whether any of the compact manifolds other than tori which admit Anosov diffeomorphisms are $T$-manifolds. Some of the nilmanifold examples definitely are not $T$-manifolds.

Finally, I would like to thank John Milnor and the referee for reformulating Theorem 3 and the Lemma and shortening their proofs.

\section{Proofs.}

Proof of Lemma. $h$ induces an algebra homomorphism from the direct sum $H / J \oplus J / J^{2} \oplus J^{2} / J^{3} \oplus \ldots$ to itself. This induced homomorphism has the same Lefschetz number. But $J^{r} / J^{r+1}$ can be identified with the $r$ th exterior power $E^{r}\left(J / J^{2}\right)$. So if $\bar{h}$ denotes the induced map $J / J^{2} \rightarrow J / J^{2}$, then $L(h)=\sum L\left(E^{r} \bar{h}\right)=\sum(-1)^{r}$ trace $E^{r} \bar{h}$ since $E^{r}\left(J / J^{2}\right)$ is concentrated 
in even or odd dimensions according as $r$ is even or odd. But trace $E^{r} \bar{h}$ equals the $r$ th elementary symmetric function of the eigenvalues $\lambda_{i}$. (This is proved by putting $\bar{h}$ in triangular form over a suitable extension field.) Hence $L(h)=\Pi\left(1-\lambda_{i}\right)$. The Lemma follows.

REMARK. The proof works over any field.

Proof of Theorem 1. Assume $f$ has no fixed points. Then $L\left(f^{*}\right)=0$ since $E^{u}$ orientable implies $\left|L\left(f^{*}\right)\right|=\operatorname{Card}(\operatorname{Fix}(f))$, see [3, p. 123]. Then some $\lambda_{i}=1$, using the Lemma with $H=H^{*}(M ; R)$ and $h=f^{*}$. Hence

$$
\operatorname{Card}\left(\operatorname{Fix}\left(f^{q}\right)\right)=\left|L\left(f^{q}\right)^{*}\right|=0 \text { for all } q>0,
$$

again using the Lemma, so $f$ can have no periodic points. This contradicts the hypothesis and proves the theorem.

Proof of Theorem 2. Either $E^{s}$ or $E^{u}$ is orientable. We may assume it is $E^{u}$, since otherwise we may replace $f$ by the Anosov diffeomorphism $f^{-1}$. Theorem 2 now follows from Theorem 1 using the fact that every Anosov diffeomorphism has a periodic point (Proposition 1.7 of [2]).

Proof of Corollary. We are given an Anosov diffeomorphism $f$ of a quotient $M=G / F$, where $F$ is a finite subgroup of a compact, connected Lie group $G$.

Case I. $E^{u}$ is orientable. By a theorem of Hopf (see [5] or [1, Chapter I]), $G$ is a $T$-manifold. A well-known easy argument shows that since $G$ is connected, $H^{*}(G ; R) \cong H^{*}(M ; R)$. Thus $M$ is a $T$-manifold and Theorem 2 implies $f$ has a fixed point.

Case II. $E^{u}$ is nonorientable. Then $f$ lifts to an Anosov diffeomorphism $f^{\prime}$ : $M^{\prime} \rightarrow M^{\prime}$ of a connected 2 -fold covering $M^{\prime}$ of $M$ with $E^{u^{\prime}}$ orientable. The following result implies, as above, that $M^{\prime}$ is a $T$-manifold. Hence $f^{\prime}$, and therefore $f$, has a fixed point, completing the proof of the Corollary.

Claim. There exists a compact, connected Lie group $G^{\prime}$ and a finite subgroup $F^{\prime}$ such that $G^{\prime} / F^{\prime}$ is diffeomorphic to $M^{\prime}$.

Proof. Let $p: G \rightarrow G / F=M$ and $p^{\prime}: M^{\prime} \rightarrow M$ be the projection maps. Set $\pi=p_{*} \pi_{1} G \cap p_{*}^{\prime} \pi_{1} M^{\prime}$ and let $p_{0}: G^{\prime} \rightarrow M$ be the covering space such that $p_{0 *} \pi_{1} G^{\prime}=\pi$. Now $G^{\prime}$ is also the covering space of $G$ associated to the subgroup $p_{*}^{-1}(\pi)$ of $\pi_{1} G$.

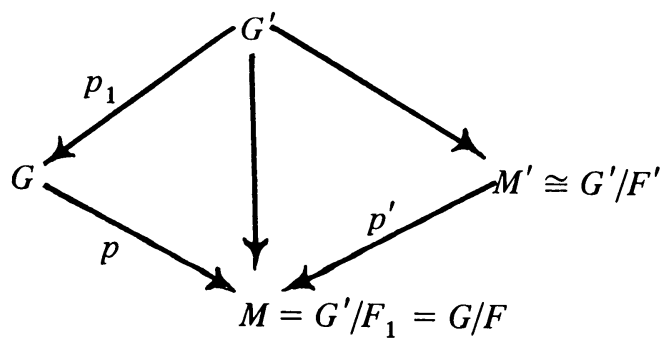

It is well known that $G^{\prime}$ is therefore a connected Lie group and the covering projection $p_{1}: G^{\prime} \rightarrow G$ is a homomorphism. Clearly $\left[\pi_{1} M: \pi\right]<\infty$, so $G^{\prime}$ is compact. 
Let $F_{1}$ denote the subgroup $p_{1}^{-1}(F)$ of $G^{\prime}$. There is a natural isomorphism $h: \pi_{1} M / \pi \rightarrow F_{1}$. Set $F^{\prime}=h\left(p_{*}^{\prime} \pi_{1} M^{\prime} / \pi\right) \subset F_{1}$. Then $F^{\prime}$ is a finite subgroup of $G^{\prime}$ and the natural projection $G^{\prime} / F^{\prime} \rightarrow G^{\prime} / F_{1}=M$ induces a monomorphism of fundamental groups which carries $\pi_{1}\left(G^{\prime} / F^{\prime}\right)$ onto $p_{*}^{\prime} \pi_{1} M^{\prime}$. It follows from the uniqueness of covering spaces associated to a subgroup that $G^{\prime} / F^{\prime}$ is diffeomorphic to $M^{\prime}$. This completes the proof.

Proof OF TheOREM 3. Suppose some eigenvalue of $\bar{f}^{*}$ is a $q$ th root of unity, $q>0$. As in the proof of Theorem 2, we may assume $E^{u}$ is orientable. Then $\operatorname{Card}\left(\operatorname{Fix}\left(f^{a}\right)\right)=\left|L\left(f^{a}\right)^{*}\right|=0$ by the Lemma, contradicting Theorem 2.

REMARK. A 2-fold cover of a $T$-manifold might not be a $T$-manifold, e.g., $S^{1} \times R P^{2}$. Thus the hypothesis of orientability in the theorems cannot be dispensed with as in the proof of the Corollary.

\section{REFERENCES}

1. A. Borel, Topics in the homology theory of fibre bundles, Lecture Notes in Math., vol. 36, Springer-Verlag, Berlin, 1967. MR 36 \#4559.

2. J. Franks, Anosov diffeomorphisms, Proc. Sympos. Pure Math., vol. 14, Amer. Math. Soc., Providence, R.I., 1970, 61-93. MR 42 \#6871.

3. Anosov diffeomorphisms on tori, Trans. Amer. Math. Soc. 145 (1969), 117-124. MR 40 \#6567.

4. M. Hirsch, Anosov maps, polycyclic groups and homology, Topology 10 (1971), 177-183. MR 44 \#1054.

5. H. Hopf, Über die Topologie der Gruppenmannig-faltigkeiten und ihrer Verallgemeinerungen, Ann. of Math. (2) 42 (1941), 22-52. MR 3, 61.

6. A. Manning, Anosov diffeomorphisms on nilmanifolds, Proc. Amer. Math. Soc. 38 (1973), 423-426. MR 49 \#8059.

7. There are no new Anosov diffeomorphisms on tori, Amer. J. Math. 96 (1974), 422-429. MR 50 \#11324.

8. M. Shub, Endomorphisms of compact differentiable manifolds, Amer. J. Math. 91 (1969), 175-199. MR 39 \#2169.

9. S. Smale, Differentiable dynamical systems, Bull. Amer. Math. Soc. 73 (1967), 747-817. MR 37 \#3598.

Institute for Defense Analyses, Princeton, New Jersey 08540 